УДК 621.317 .328

\title{
ПРИМЕНЕНИЕ АДАПТИВНОГО АЛГОРИТМА ПРИ РАЗРАБОТКЕ ЦИФРОВОЙ ЭЛЕКТРОМАГНИТНОЙ МОДЕЛИ МЕСТНОСТИ
}

\author{
Звездина М.Ю., Шокова Ю.А., Кривцова М.Г. \\ ФГБОУ ВО «Донской государственный технический университет», \\ Ростов-на-Дону, е-mail: zvezdina_m@mail.ru
}

\begin{abstract}
Широкое применение систем связи привело к необходимости создания цифровых электромагнитных моделей местности. Данные модели основаны на объединении цифровых карт местности и результатов визуализации распределения плотности потока энергии от объектов излучения, позволяют осуществлять мониторинг электромагнитной обстановки. Разработка электромагнитных моделей местности связана с существенными вычислительными затратами. Для их сокращения предлагается наряду с использованием плоскостных распределений плотности потока энергии применять адаптивный алгоритм выбора шага координатной сетки. Алгоритм базируется на учете электродинамических эффектов, обусловленных конструкцией антенны и местом ее размещения. Вычислительный эксперимент по расчету распределения плотности потока энергии для зеркальной антенны показал, что предложенный алгоритм выбора расчетных точек позволяет снизить объем вычислений на $20 \%$.
\end{abstract}

Ключевые слова: электромагнитная модель местности, распределение плотности потока энергии, визуализация результатов, адаптивный алгоритм выбора

\section{ADAPTIVE ALGORITHM FOR DIGITAL ELECTROMAGNETIC TERRAIN MODEL DEVELOPMENT \\ Zvezdina M.Yu., Shokova Yu.A., Krivtsova M.G. \\ Don State Technical University, Rostov-on-Don, e-mail: zvezdina_m@mail.ru}

\begin{abstract}
The extensive use of communications has resulted in the necessity of digital electromagnetic terrain model development. Models are based on joining digital terrain maps and visualization results for energy flux density distribution of radiation bodies; they promote electromagnetic environment monitoring. Model developing has high computational costs. It is proposed to use adaptive algorithm of coordinate grid step size control as well as plane energy flux density distributions to reduce the costs. The adaptive algorithm is based on consideration of electromagnetic effects caused by antenna construction and placement. Simulation experiment on energy flux density estimation for reflector antenna has showed that the proposed solution decreases computational cost up to $20 \%$.
\end{abstract}

Keywords: electromagnetic terrain model, energy flux density distribution, visualization of results, step control adaptive algorithm

Переход к глобальному информационному обществу, заложенный в государственной программе «Информационное общество (2011-2020 годы)» [6], определил в качестве основного направления развития экономики переход к информационно-коммуникационной инфраструктуре в системе общественного производства. Следствием данного перехода является расширение областей применения инфокоммуникационных технологий, в том числе систем широкополосного доступа, обеспечивающих в том числе мобильный доступ в Интернет. В результате размещения излучающих средств на территории жилой застройки существенно ухудшилась экологическая обстановка по электромагнитному фактору [1], что, в свою очередь, вызвало необходимость в визуализации электромагнитной остановки, создаваемой телекоммуникационными техническими средствами, расположенными на больших территориях [2]. Результатом такой процедуры, как показано в [1-2], является формирование цифровой электромагнитной модели местности на основе объединения цифровой карты местности с результатами визуализации распределения плотности потока энергии (ППЭ) от объектов излучения. При этом основной проблемой является необходимость выполнения большого объема вычислений, обусловленных необходимостью построения подробной карты на больших территориях.

Цель статьи - оценка возможности снижения вычислительных затрат в процессе визуализации распределения плотности потока энергии от объектов излучения путем построения адаптивного алгоритма выбора числа и мест размещения расчетных точек.

Таким образом, рассматриваемые в статье вопросы являются актуальными как при непосредственной разработке новых цифровых моделей, так и в процессе обучения их созданию специалистов в области радиотехники.

\section{Этапы разработки цифровой модели электромагнитной обстановки}

Разработка цифровой модели электромагнитной остановки, как показано в [1], 
базируется на использовании геоинформационных систем и предполагает три основных этапа: инвентаризацию излучающих объектов с соответствующей классификацией по степени экологической опасности; создание на цифровой карте местности слоя с размещением излучающих объектов; нанесение слоя с распределением плотности потока энергии от источников на карту. При этом основные вычислительные затраты приходятся на разработку базы данных по типовым источникам излучения, находящимся на исследуемой местности. В связи с этим выполним более подробный анализ операций, выполняемых на данном этапе проектирования.

Анализ начнем с выбора метода организации электромагнитного мониторинга на больших территориях. Для оценки эффективности метода сформулируем требования к используемым на данном этапе методам. На наш взгляд, к ним можно отнести: возможность привязки результатов к цифровой карте местности; получение информации для каждого участка местности, включая наземные и воздушные; высокую оперативность обновления информации; относительно невысокие вычислительные затраты на визуализацию результатов.

С учетом перечисленных выше требований выполненный анализ известных методов, приведенных в работах $[1,2]$, показал, что при разработке цифровой модели электромагнитной обстановки местности можно использовать два основных метода, дополняющих друг друга - инструментальный и метод расчетного прогнозирования. Сравнительный анализ данных методов показал, что на больших территориях применение инструментальных методов является неэффективным по двум основным причинам: возможности измерений только на отдельных приземных участках и снижении оперативности обновления информации изза больших площадей территории. Метод расчетного прогнозирования, базирующийся на использовании электродинамических моделей, в отличие от инструментального метода, для больших территорий обеспечивает возможность получения практически неограниченного количества информации о пространственно-временном распределении электромагнитных полей в режиме реального времени. Однако его основным недостатком, вытекающим из привлечения электродинамической теории, является создание сложных в теоретическом плане электродинамических моделей.

\section{Требования к программному обеспечению}

Выбор программного обеспечения для расчета плотности потока энергии от излучающих радиотехнических объектов необходимо осуществлять с учетом особенностей применения методов расчетного прогнозирования, в частности необходимо определить требования к программам визуализации электромагнитного поля. В общем случае данные требования можно объединить в следующий перечень. Во-первых, в обязательном порядке должен отображаться уровень

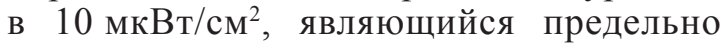
допустимым для излучающих объектов в СВЧ диапазоне в соответствии с [5] и определяющий границы санитарно-защитных зон для антенн базовых станций. Во-вторых, разработка программы должна осуществляться с использованием методов расчетного прогнозирования на основе применения математических моделей, учитывающих электродинамические эффекты, т.е. конструкцию антенны и место её установки. В-третьих, распределение ППЭ вблизи места установки антенны должно отображаться на рельефных графиках в двух сечениях - горизонтальном и вертикальном. Положение сечений относительно фазового центра антенны должно задаваться. В-четвертых, реализация программы должна быть выполнена в программном продукте, на который имеется лицензия у вуза. В-пятых, требования к программному оборудованию не должны быть слишком высокими, поскольку парк ЭВМ в учебных заведениях не обладает большими мощностями. Два последних требования добавляются при использовании программных средств в учебном процессе вуза.

Анализ известных программ, разработанных для визуализации электромагнитной обстановки вблизи места размещения излучающих антенн в диапазоне СВЧ, показал, что наиболее эффективными являются программные продукты SanZone [7] и RPS2 [8]. Однако данные программы являются коммерческими с закрытыми кодами. Вследствие этого не могут быть использованы в учебных целях при разработке цифровой электромагнитной модели местности. Кроме того, данные модели не учитывают место установки антенны. Альтернативным вариантом в учебном процессе является разработка самостоятельных программ в пакете MATLAB. 


\section{Анализ методики расчета распределения ППЭ}

для типовых элементов базы данных

В работе [1] было показано, что основными источниками преднамеренного излучения являются радиотехнические системы, к которым в городских условиях относятся эфирное телевидение, радиовещание, а также системы сотовой и транкинговой связи. Кроме того, к данным источникам можно также отнести антенны радиорелейной линии передач и систем спутниковой связи. При разработке электродинамической модели необходимо, как отмечалось ранее, учитывать конструкцию антенны, место ее размещения, частотный диапазон ее функционирования. В перечисленных выше радиотехнических системах наиболее широко применяются панельные излучатели, а также апертурные антенны (зеркальные и рупорные). Алгоритм расчета величины ППЭ для каждого из перечисленных выше типов антенн приведен в [5]. Выполним анализ данного алгоритма с целью выявления операций, на которых возможно осуществить сокращения вычислительных затрат.

«Методические указания...» [5] независимо от типа конструкции антенны включают два этапа: привязку системы отсчета к фазовому центру антенны с переходом к сферической системе координат; размещение расчетных точек в пространстве вблизи антенны. Второй из перечисленных выше этапов связан с выбором положения центров $M\{x, y, z\}$ расчетных точек. При этом решаются два вопроса: выбор представления размерности решения - $3 D$ (в объеме) или $2 D$ (на плоскости); выбор типа координатной сетки размещения центров расчетных точек - равномерный или адаптивный.

Анализ выполняемых операций показал, что сокращение объема вычислений можно обеспечить только на втором этапе. Достигается это, во-первых, выбором представления решения на плоскости с визуализацией в виде графика уровней. Наиболее эффективными для анализа электромагнитной обстановки являются сечения в главных плоскостях. При этом при задании высот сечений горизонтальными плоскостями наиболее эффективными для анализа являются два среза. Первый из них проходит на высоте расчета санитарно-защитной зоны (2 метра от поверхности Земли или на высоте последнего этажа высотной застройки при размещении антенны на крыше) [5]. В вертикальной плоскости сечения целесообразно проводить через фазовый центр антен- ны. Здесь в обязательном порядке должно анализироваться сечение, проходящее через фазовый центр антенны и направление максимального излучения антенны. Данное сечение, относящееся к наихудшему варианту событий, обычно используется для оценки электромагнитной обстановки на различных этажах соседних с антенной зданий.

Во-вторых, сокращение объема вычислений на втором этапе может быть достигнуто путем использования выбора координатной сетки с адаптивным шагом размещения. При этом учет особенностей распространения электромагнитных волн от источника позволяет рекомендовать выбор наиболее мелкого шага дискретной сетки, должен быть использован внутри границы санитарно-защитной зоны (вблизи антенны), а более крупный - вне ее. Таким образом, неисследованным остается вопрос разделения пространства вокруг антенны на области с различным шагом размещения центров расчетных точек.

\section{Алгоритм разделения пространства вокруг антенны на области \\ с различным шагом размещения центров расчетных точек}

Алгоритм разделения пространства вокруг антенны на области с различным шагом размещения центров расчетных точек связан с учетом электродинамических особенностей конструкции антенны и места ее установки. В «Методических указаниях ...» [5] для типовых конструкций антенн систем сотовой связи можно выделить три модели разделения пространства вокруг антенны на характерные области: для антенн УКВ диапазона при размещении на крыше здания; для зеркальных антенн и для рупорных антенн. Геометрии разделения пространства для первых двух моделей приведены соответственно на рис. 1, a, б. В третьей модели в силу особенности конструкции антенны пространство разделяется на два полупространства - переднее (куда направлено излучение) и заднее.

При использовании первой модели направленные свойства антенны, размещаемой в точке $\Phi$, описываются паспортными данными в виде диаграммы направленности в главных сечениях, а само пространство в соответствии с теорией геометрической оптики разделяется на три области. Данные области включают: область прямого луча I, область II, в которой поле определяется суммой прямого и отраженного луча, а также область тени III. С учетом описанного 
разделения пространства для первой модели в качестве области, где можно использовать более крупный шаг координатной сетки, следует рассматривать область тени III. Как несложно заметить из анализа рис. 1, a, а также результатов моделирования структуры ППЭ, приведенных, например, в работе [4], данная область занимает достаточно большую область, что позволит существенно сократить вычислительные затраты.

Вторая модель является более сложной по разбиению пространства. Это связано со способом формирования излучаемого потока энергии - через рефлектор (зеркало). В электродинамической модели это отражается необходимостью учета дифракционных явлений на кромках зеркала, приводящих к переотражению поля облучателя в переднее полупространство и затеканию в заднее полупространство. В результате пространство вокруг зеркальной антенны разбивается на пять характерных областей: Среди них, как показано на рис. 1, б, выделяют область I главного лепестка и близких к нему боковых лепестков диаграммы направленности; область дифракционного поля II; область переднего полупространства III, где наблюдается и дифракционное поле от кромок зеркала; область IV, где сосредоточены дальние боковые лепестки диаграммы направленности; прожекторная область V, где наблюдается максимальное поле антенны.

Анализ структуры поля в выделенных областях, описанный в [5], а также результаты моделирования структуры распределения ППЭ, приведенные в работах $[3,4,9,10]$, показывают, что в областях III и IV шаг координатной сетки может быть выбран большим требуемого в 0,25 длины волны для получения точных результатов, в областях V и I равным данной величине, а в области II - меньшим 0,25 длины волны. После более детальных исследований можно уточнить размеры исследуемой области в переднем и заднем полупространстве вблизи зеркальной антенны.

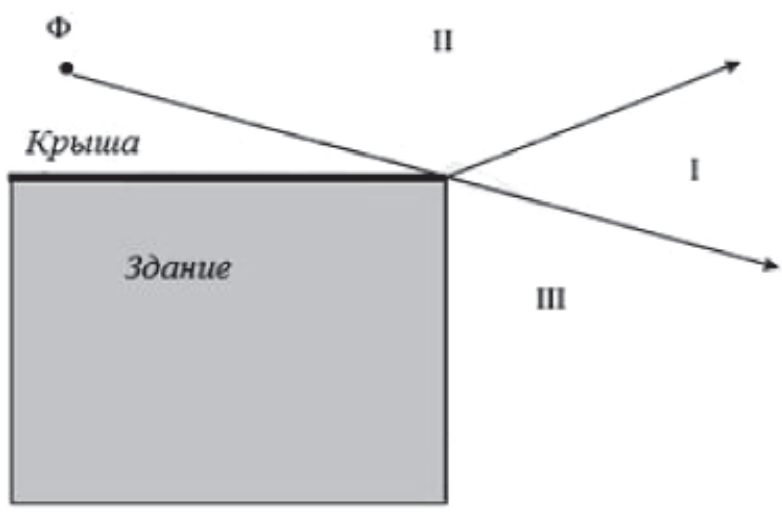

a

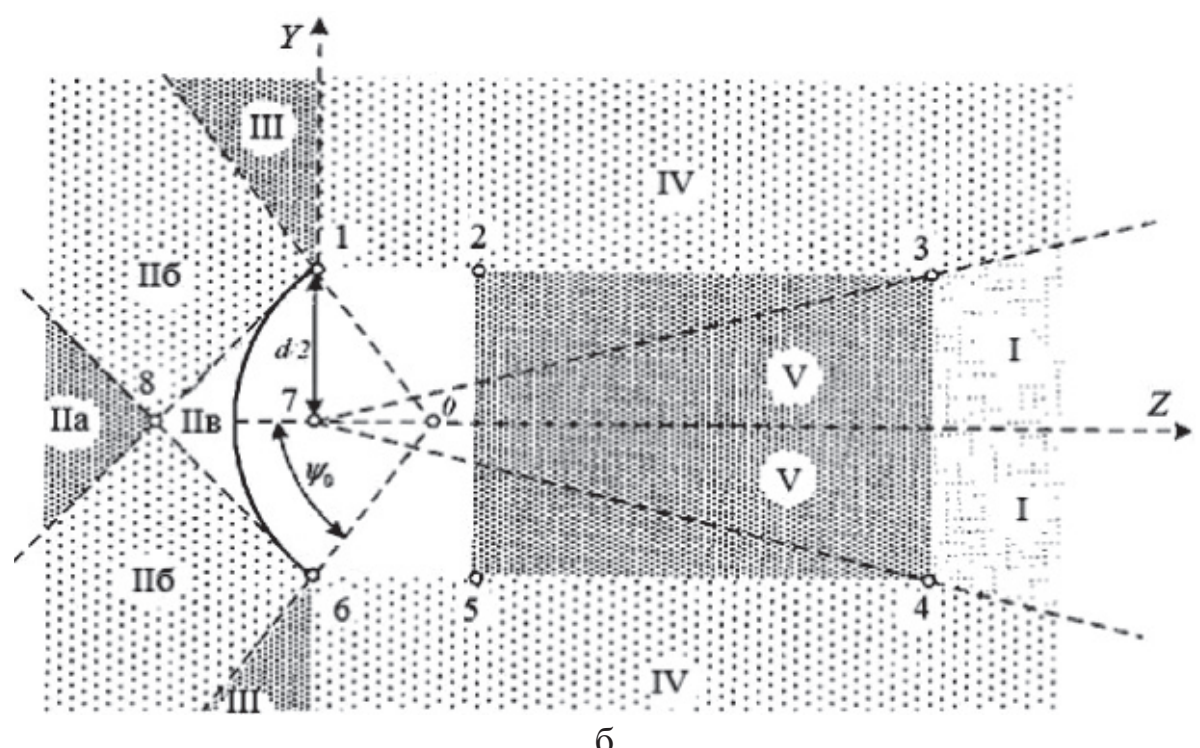

б

Рис. 1. Разделение пространства вблизи конструкиии антенны на области 
Для третьей модели в качестве рекомендации можно предложить в переднем полупространстве использовать более мелкую сетку, а в заднем - более крупную сетку.

\section{Анализ результатов моделирования}

Оценим сокращение вычислительных затрат на примере нахождения распределения ППЭ в горизонтальной плоскости для зеркальной антенны с диаметром круглой апертуры в 1,44 м, углом полураскрыва в 90 градусов, коэффициентом направленного действия в 30 дБ. Частота передатчика составляла 6 ГГц, а его мощность - 100 Вт. Фазовый центр антенны размещался на высоте $H_{A}=7 \mathrm{~m}$ от поверхности Земли. Визуализацию электромагнитной обстановки будем производить на высоте максимального излучения антенны $H_{T}=H_{A}=7 \mathrm{~m}$. Вычислительные затраты для такой конструкции антенны в зависимости от выбираемого шага координатной сетки, а также ее типа приведены в таблице. Выбор шага при адаптивном алгоритме осуществлялся с использованием сформулированных выше рекомендаций.
На рис. 2, а, б приведены распределения ППЭ в горизонтальной плоскости для двух типов координатной сетки равномерной с шагом 0,25 м и адаптивной с минимальным шагом 0,1 м и максимальным шагом в 0,25 м. Величина ППЭ показана на линиях уровней. Штриховые линии иллюстрируют границы характерных областей.

Анализ данных рисунков показывает, что точность получаемых результатов, особенно в области заднего полупространства (область II), существенно зависит от типа координатной сетки и величины ее шага. Применение адаптивного алгоритма обеспечивает не только получение более точной визуализации, но позволяет почти на $20 \%$ сократить вычислительные затраты.

\section{Выводы}

Выполненный анализ операций, необходимых для разработки цифровой электромагнитной модели местности, показал, что основной трудоемкой операцией является создание для объектов излучения базы данных, содержащих визуализацию

Вычислительные затраты в зависимости от выбора типа координатной сетки

\begin{tabular}{|c|c|c|c|c|c|}
\hline \multirow{3}{*}{ Параметры } & \multicolumn{5}{|c|}{ Шаг координатной сетки } \\
\hline & \multicolumn{4}{|c|}{ Равномерная сетка } & Адаптивная сетка \\
\hline & $1 ? 5 \mathrm{M}$ & $0,5 \mathrm{~m}$ & $0 ? 25 \mathrm{M}$ & $0,1 \mathrm{M}$ & 0,25 и 0,1 м \\
\hline Число расчетных точек & 982 & 1997 & 8070 & 41818 & 34192 \\
\hline Время вычислений, с & 24,3 & 32,9 & 72,67 & 375,4 & 292 \\
\hline
\end{tabular}

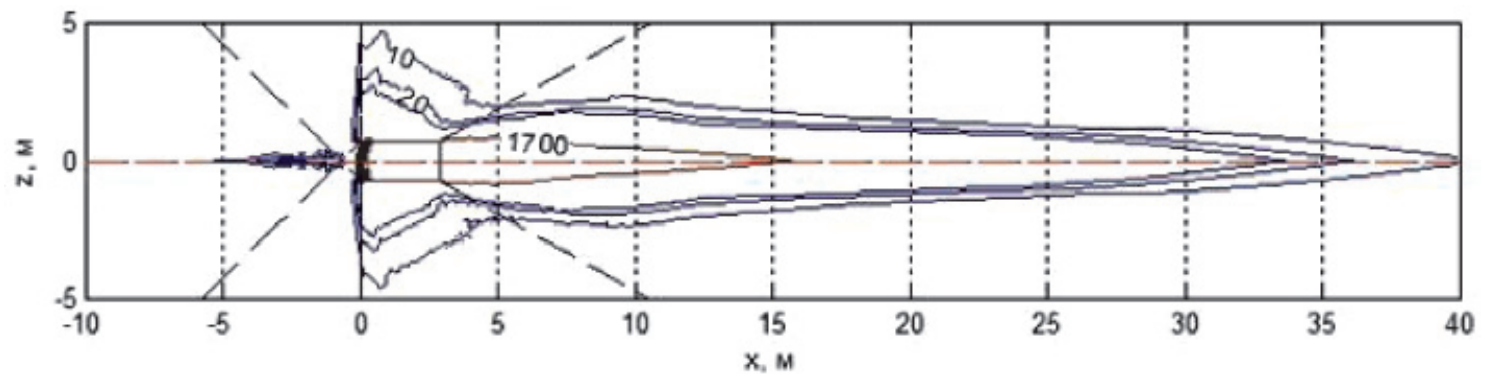

a

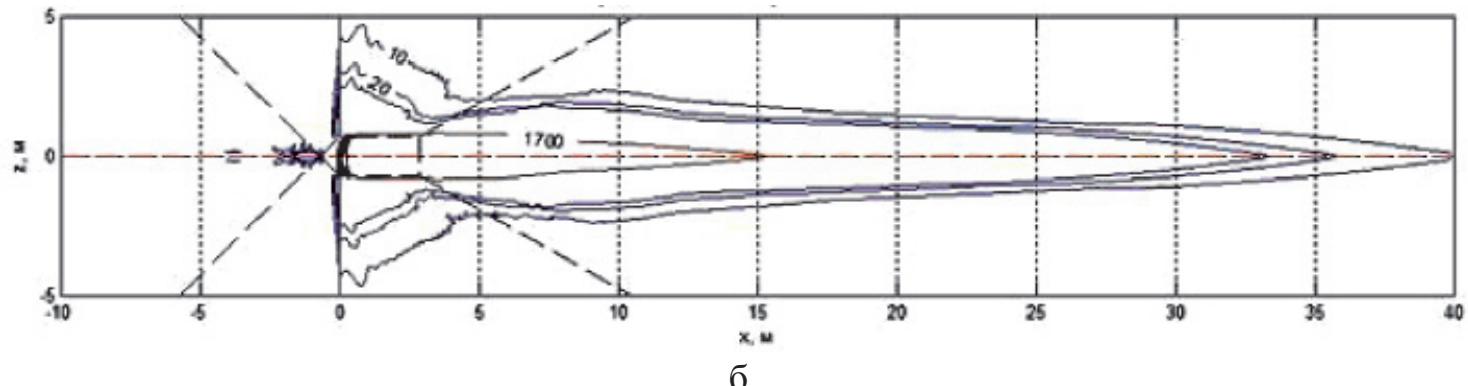

Рис. 2. Зависимость вида распределения ППЭ от типа координатной сетки 
распределения плотности потока энергии в пространстве вблизи антенн. Показано, что применение метода расчетного прогнозирования, базирующегося на использовании теории электродинамики для описания явлений излучения и дифракции, позволяет учесть как конструкцию антенны, так и место ее размещения. Выполненный анализ известного программного обеспечения показал, что существующие программные продукты выполнены с использованием закрытых кодов и не могут быть применены в процессе обучения для привития навыков разработки цифровых карт. В связи с этим в качестве программного продукта для визуализации распределения ППЭ объектов излучения предложено использовать пакет MATLAB. Для сокращения объема вычислений при создании базы данных объектов излучения предложен адаптивный алгоритм, позволяющий на основе учета электродинамических эффектов, обусловленных особенностями конструкции антенны и места ее расположения, формировать рекомендации по использованию адаптивного шага размещения расчетных точек. Выполненный на примере зеркальной антенны вычислительный эксперимент показал, что использование предложенного адаптивного алгоритма позволяет сократить объем вычислений на $20 \%$.

\section{Список литературы}

1. Довбыш В.Н., Сивков В.С. Цифровая электромагнитная модель местности // Инфокоммуникационные технологии. -2007 . - Т. 5, № 1. - С. $85-88$.

2. Довбыш В.Н., Сивков В.С., Сподобаев Ю.М. Визуализация электромагнитной обстановки, создаваемой телекоммуникационными техническими средствами, распо- ложенными на больших территориях // Антенны. - 2006. № $10 .-$ C. $58-62$

3. Звездина М.Ю., Шокова Ю.А., Шоков А.В. Визуализация электромагнитного поля апертурной антенны подвижной связи в рамках социально ориентированного мониторинга // Universum: Технические науки - 2015, - № 8 [Электронный ресурc]. - URL: http://7universum.com/ru/tech/ archive/item/ 2590 (дата обращения 9.09.2016).

4. Звездина М.Ю., Шокова Ю.А., Шоков А.В. Социально ориентированный мониторинг окружающей среды // Применение инноваций при разработке радиотехнических систем. - М.: Изд. Дом «Академия Естествознания», 2015. - C. 44-69.

5. МУК 4.3.1167-02. Методические указания по определению плотности потока мощности электромагнитного поля в местах размещения радиосредств, работающих в диапазоне частот 300 МГц - 300 ГГц. Утверждены Председателем Госкомитета санитарно-эпидемиологического надзора РФ 7 октября 2002 года. Представлены Минсвязи России № HTУ-1/237 от 20.04.01 г.) [Электронный ресурс]. - URL: http://bestpravo.ru/rossijskoje/so-instrukcii/t5r.htm. (дата обращения: 20.04.2016 г.)

6. Постановление Правительства РФ от 15.04.2014 № 313 (ред. от 17.06.2015) «Об утверждении государственной программы Российской Федерации «Информационное общество (2011-2020 годы)» [Электронный ресурс]. - URL: http://www.consultant.ru/document/cons_doc_LAW_162184/ (дата обращения: 2.09.2016 г.)

7. Программа SanZone [Электронный ресурс]. - URL: http://www.ing-tv.ru/index/sanzone/0-53 (дата обращения: 09.09.2016).

8. RPS2: Radio Planning System 2. [Сайт Центра компьютерных технологий «Связь Телеком Софт»] [Электронный pecypc]. - URL: http://www.rps2.ru/ (дата обращения: 06.09.2016 г.).

9. Visual representation of energy flux density in the backspace of circular aperture reflector antenna for mobile communications / M.Yu. Zvezdina et all // International Science Journal Theoretical \& Applied Science. - 2015. - № 8 (28). P. 56-64; doi: 10.15863/TAS.2015.08.28.8 (дата обращения 09.09.2016 г.)

10. Zvezdina M.Yu., Shokova Yu.A., Shokov A.V. Visual representation characteristics of calculation results in laboratory research on electromagnetic ecology // Proc. of 9th International Conf. On Application of Information and Communication Technologies (AICT 2015). 14-16 October 2015, Rostov-onDon, Russia. IEEE Proc. AICT-2015. - P. 399-403. doi: 10.1109/ ICAICT.2015.7338588. 